\title{
AN OVERVIEW OF INTEGRATION METHODS FOR HYPERSINGULAR BOUNDARY INTEGRALS*
}

\section{Earlin Lutz}

486 Engineering and Theory Center

Comell University

Ithaca, NY 14850

$$
\text { L.J. Gray }
$$

Mathematical Sciences Section Engineering Physics and Mathematics Division

Oak Ridge National Laboratory

P. O. Box 2008, Bldg. 6012

Oak Ridge, TN 37831-6367

\section{Anthony R. Ingraffea}

\author{
317 Hollister Hall \\ Cornell University \\ Ithaca, NY 14850
}

\section{DISCLAIMER}

\begin{abstract}
This report was prepared as an account of work sponsored by an agency of the United States Government. Neither the United States Government nor any agency thereof, nor any of their employees, makes any warranty, express or implied, or assumes any legal liability or responsibility for the accuracy, completeness, or usefulness of any information, apparatus, product, or process disclosed, or represents that its use would not infringe privately owned rights. Reference herein to any specific commercial product, process, or service by trade name, trademark, manufacturer, or otherwise does not necessarily constitute or imply its endorsement, recommendation, or favoring by the United States Government or any agency thereof. The views and opinions of authors expressed herein do not necessarily state or reflect those of the United States Government or any agency thereof.
\end{abstract}

* This research was sponsored by the Applied Mathematical Sciences Research Program, Office of Energy Research, U.S. Department of Energy under contract DE-AC05-84OR21400 with the Martin Marietta Energy Systems, Inc. 


\title{
An Overview of Integration Methods for Hypersingular Boundary Integrals
}

\author{
Earlin Lutz \\ 486 Engineering and Theory Center \\ Cornell University \\ Ithaca $N Y$ 14850 USA \\ Leonard J. Gray \\ Mathematical Sciences Section \\ Oak Ridge National Labs \\ Oak Ridge TN 37831 USA \\ Anthony R. Ingraffea \\ 317 Hollister Hall \\ Cornell liniversity \\ Ithaca $N Y 14850$ USA
}

\begin{abstract}
Several methods of analyzing the hypersingular gradient BIE have been deveioped recently. This paper is a review highlighting the numerous common aspects and several differences among the methods. Significant common aspects include (a) a regularization of constant and linear terms, (b) analysis of integration points near rather than on the surface, and (c) analysis of the neighborhood of the singular point rather than of individual elements.
\end{abstract}

\section{Introduction}

Singular boundary integral equations have been used since the middle 1960's for solution of boundary value problems such as potential flow, wave scattering, and elasticity. Historically, there are three phases in the development of this technology. First, theoretical analysis of singular integrals began prior to the computer era. This style of analysis ([CZZ2], [Mik65], [MP80], [Kup65]) is known as generalized potential theory and is still present today in the 'indirect' formulations 
of boundary integrals. Second, engineering researchers in the 1960's ([Riz69], [Cru69], [Jas63]) modified the formulation so that the abstracc boundary integral would make direct use of true 'physical unknowns' (e.g. displacements and tractions in elasticity problems) rather than the elusive 'surface densities' used in the earlier indirect forms.

Third, a particular style of impelementation was developed ([LW76], [BB81], [BTW84], [BD88]) in the 1970's and 1980's by borrowing interpolation technology from the finite element method. This particular blend of theory and implementation is known as the 'boundary element method' (BEM). From an end-user's view, this method is competetive with (i.e. has advantages for some problems, disadvantages for others) the finite element and finite difference methods as a day-to-day engineering tool.

In the late 1980's, several methods have been developed to deal with the gradient (derivative) the boundary integral for the BEM. This integral contains 'hy'persingular' terms whose behavior was not previously understood. This paper is a review highlighting the numerous common aspects and several differences among these methods. Significant common aspects include (a) a regularization of constant and linear terms, (b) analysis of integration points near rather than on the surface, and (c) analysis of the neighborhood of the singular point rather than of individual elements.

\section{Organization}

Section 1 introduces the hypersingular integration problem in the BEM. Section 2 gives the notation and terminology for the BIE and its gradient, and identifies several points that are in common to all methods of analyzing the hypersingular problem. Section 3 describes how the various methods diffe: from one another.

To simplify the presentation, mathematical examples are given only for the Laplace equation, with occasional reference to elasticity. A complete set of analogous forms for elasticity is found in [Lut91].

\section{The Singular Integration Problem}

From a programmer's view, the boundary element method presents challenging numerical problems associated with computation of singular integrals, i.e. integrals in which the integrand is unbounded at certain points in the domain of integration. This problem is resolved in the primary BIE by either (a) a Cauchy principal value analysis or (b) a rigid body motion argument.

Analysis of (a) the elastic fieid around a crack, (b) the electric field ar zund a thin non-conducting sheet in a conducting medium, and (c) acoustic wave scattering in a medium with a thin rigid inclusion are all characterized by a single surface placed so that both of its (oriented) sides are incident to the same physical region. Sign changes in the in tegrands at mated points on opposite sides of the feature lead to cancellation of terms describing normal gradients of the 
solution fields (respectively, tractions, normal fluxes, and normal velocities) in the primary BIE.

An established remedy for this degeneracy is to subdivide the geometry into multiple regions so that opposite sides of the thin feature appear in different regions ([BIL_1], [JSR90]). This is effective, but introduces additional geometric processing not present in pure boundary-only methods. An alternative is to seek some other boundary integral in which the solution functions on the singular surface are fully represented.

Several researchers (e.g. [Bui77], [Wea77], [PCH 87], [IP88], [RB90], [CN90], [KSRR90], [GM190], [Lut91] ) have aittempted to resolve this difficulty by applying some form of the the derivative (gradient) of the original BIE. Differentiation raises the degree of singularity in the integrands. The Cauchy singularity of the primary BIE becomes an apparentl $j$-more-difficult 'hypersingular' term, and new instances of the Cauchy type of singularity appear. An early analysis addressing many issues not resolved until the current time is found in ([CVB71]).

Cursory reading of the various analyses might leave one with the impression that there are significant differences among the assumptions, methods, and sometimes-complicated results. This is not the case. This paper is an overview that attempts to emphasize the common aspects of the various approaches.

\section{The Boundary Integral Equation}

This section gives the notation for the Laplace BIE and then identifies issues that are common to all analyses of the gradient BIE.

The primary boundary integral equation says that for any function that satisfies $\nabla^{2} u=0$ the solution values $u(\mathbf{x})$ for any point not on the boundary $S$ of a region (see Fig. 1(a)) can be defined exactly by the following integral that involves only (a) the boundary $S$, (b) the solution values $u(\mathbf{y})$ and $\frac{\partial u(\mathbf{y})}{\partial \mathbf{n}(\mathbf{y})} S$, and (c) a kernel function $G$ and its normal derivative $H$, in the form

$$
\gamma(\mathbf{x}, S) u(\mathbf{x})=\int_{S} G(\mathbf{x}, \mathbf{y}) \frac{\partial u(\mathbf{y})}{\partial \mathbf{n}(\mathbf{y})}-H(\mathbf{x}, \mathbf{y}, \mathbf{n}) u(\mathbf{y}) d S(\mathbf{y})
$$

or, omitting references to geometric parameters $\mathbf{x}$ and $\mathbf{y}$

$$
\gamma u=\int_{S} G \frac{\partial u}{\partial \mathrm{n}}-H u d S
$$

The kernels $G$ and $I I$ are defined by

\begin{tabular}{|c|c|c|}
\hline & $2 \mathrm{D}$ & $3 \mathrm{D}$ \\
\hline$G$ & $\frac{1}{4 \pi} \ln \frac{1}{r}$ & $\frac{1}{4 \pi r}$ \\
\hline$H=\frac{\partial G}{\partial \mathbf{n}(\mathbf{y})}$ & $-\frac{1}{4 \pi} \frac{\mathbf{r} \cdot \mathbf{n}}{r^{2}}$ & $-\frac{1}{4 \pi} \frac{\mathbf{r} \cdot \mathbf{n}}{r^{3}}$ \\
\hline
\end{tabular}


where $\mathbf{r}=\mathbf{y}-\mathbf{x}, r=|\mathbf{r}|$ and $\mathbf{n}=\mathbf{n}(\mathbf{y})$ is the outward surface normal at $\mathbf{y}$.

The coefficient $\gamma(\mathbf{x}, S)$ is 1 for any interior point and 0 for any exterior point. Some types of singularity analysis define values of $\gamma$ when $\mathbf{x}$ is exactly on the boundary, e.g. at surface point $\bar{x}$. For the Laplace equation, this boundary value is exactly the included angle of the surface at the singular point. Other analyses leave $\gamma$ 'undefined' on the boundary and instead produce expressions for (different) limiting values of the integrals when $x$ approaches point $\bar{x}$ from either the interior or exterior.

Taking partial derivatives of (1) with respect to a coordinate of $\mathbf{x}$ produces a corresponding boundary integral for partial derivatives of $u$ :

$$
\gamma \frac{\partial u(\mathbf{x})}{\partial x_{j}}=\int_{S} \frac{\partial G}{\partial x_{j}} \frac{\partial u}{\partial \mathbf{n}}-\frac{\partial H}{\partial x_{j}} u d S
$$

The kernels $H, \frac{\partial G}{\partial x_{j}}$, and $\frac{\partial I}{\partial x_{j}}$ in (2) and (1) are derived from $G$ by a sequence of differentiations, as in Fig. 2. As differentiation raises the singularity of the kernel, computations for points $\mathbf{x}$ that are approaching a surface point $\overline{\mathbf{x}}$ (see Fig. 1(a)) become increasingly difficult.

When dealing with discretized surface functions, in tegrations are carried out only over some small portion of the surface. In subsequent sections, $S_{1}$ always denotes this local patch (Fig. 1(b)).

\section{$2.1 \quad$ Regularization}

All analyses of the gradient BIE depend on isolation of the various degrees of singularity. This is done by a regularization step as follows. Write the potential function in the neighborhood of a surface point $\bar{x}$ as

$$
u(\mathbf{y})=\bar{u}+\overline{a_{i}}\left(y_{i}-\overline{x_{i}}\right)+w(y)
$$

where $\bar{u}$ and the $\bar{a}_{i}$ are the potential and fluxes at $\bar{x}$ itself and $w(\mathbf{y})$ contains residual terms of order $O\left(|\mathbf{y}-\overline{\mathbf{x}}|^{2}\right)$. Because (3) is a Cartesian expansion (not just a surface function), it can be differentiated to obtain

$$
\frac{\partial u(\mathbf{y})}{\partial y_{j}}=\delta_{i j} \overline{a_{i}}+\frac{\partial w(\mathbf{y})}{\partial y j}
$$

where $\frac{\partial w(\mathbf{y})}{\partial x_{j}}$ is of order $O(r)$. becomes

Applying the series expansion, the gradient BIE (2) over surface patch $S_{1}$

$$
\delta_{i j} \bar{a}_{i}+\omega(\mathbf{x})=-\pi \int_{S_{1}}-\frac{\partial I}{\partial x_{j}} d S
$$




$$
\begin{aligned}
& +\sqrt{a_{i} S_{S_{1}} \frac{\partial G}{\partial x_{j}} \delta_{i j} n_{i} \frac{\partial H}{\partial x_{j}}\left(y_{i}-\overline{x_{i}}\right) d S} \\
& +\int_{S_{1}} G \frac{\partial w}{\partial y_{i}} n_{i}-\frac{\partial H}{\partial x_{j}} w d S
\end{aligned}
$$

(5) and (6) are the difficult (more than weakly singular) terms that require special analysis. An important aspect of $(6)$ is that the in tegral involves both $\frac{\partial G}{\partial x_{j}}$ and the hypersingular $\frac{\partial H}{\partial x_{j}}$ kernel multiplied by a linear factor. This is in contrast to the standard implementation practice of treating each kernel (multiplied by a surface function) as an independent integration problem.

The following paragraphs outline several points where integration of the gradient BIE requires revision of the standard way of organizing BIF computations.

Domain of integration Except for the Cauchy singularity, integrations of the primary BIE can proceed 1 element at a time. That is, in Fig. 1(b), in tegrals for $\overline{\mathbf{x}}$ can be done separately over the various incident elements. However, the boxed singular expressions (5) and (6) are well defined only over the entire surrounding neighborhood $S_{1}$ around $\mathbf{x}$; there is a single value for all of $S_{1}$ in the figure.

Surface Values versus Limiting Values Another feature common to all analyses is that they never allow the singular point to actually be placed on the surface. Point $\mathbf{x}$ of (1) is always considered to be close to some surface point $\overline{\mathbf{x}}$ (Fig. 1(a)). Each method provides some argument for existence of a finite limit.

When dealing with cracks, both directions of approach are significant. A point approaching one side of a crack will appear to be on the inside of (oriented) elements on that crack face and outside of oppositely oriented elements on the opposing face.

Continuity Requirements and the Cartesian Series Expansion The use of a series expansion for both constant and linear terms imposes a difficult continuity requirement, particularly at corners. The reader may consult the references for examples of the creative methods that have been applied to achieve this continuity in the absence of simple $C^{1}$ element formulations.

Use of the Cartesian (rather than parametric) series does not preclude the usual independent parametric interpolation of potential and flux. However, it does require that the implementation be able to transform the parametric expansions into Cartesian forms of the appropriate continuity at any surface point where the gradient BIE is to be computed. 


\section{Summary of Methods}

This section summarizes the general strategies that are used in various analyses of hypersingular integrals. Fig. 3 shows a classification of approaches. $A$ t the top of the decision tree, there is an initial decision as to whether the various kernels in the integrals will be (a) expanded into their (sometimes lengthy) algebraic forms or (b) left in generalized form.

The left side of the tree leads to methods based on manipulating the algebraic expansions. The crucial aspect of all these methods is to include explicitly the distance of $\mathbf{x}$ to the surface point $\bar{x}$ in the initial statement of the integral. This makes the algebra more complicated than a surface-only expansion, but the limiting values of the integrals turn out to be well defined as the distance vanishes. There is a lower level branch based on whether or not the algebraic manipulations are done in the context of an 'exclusion zone' on the surface.

Methods in the right branch of the tree do not expand the kernels. Instead, they invoke special properties that the kernels posess because of their special relationship to the differential equation. The ability to achieve convincing results by this generalized reasoning is an illustration of the remarkable power of Green's functions.

\subsection{Modal Solution Method}

The modal method is a generalized analysis that produces extremely concise results. By considering (as isolated problems) (a) a globally constant potential solution mode $u(\mathbf{x})=\bar{u}$ and then (b) a globally linear potential solution mode $u(\mathbf{x})=a_{i}\left(x_{i}-\overline{x_{i}}\right)$, it can be shown ([Lut91]) that

$$
\int_{S_{1}} \frac{\partial H}{\partial x_{j}} d S=-\int_{S_{2}} \frac{\partial H}{\partial x_{j}} d S
$$

and, for each $i$ and $j$,

$$
\begin{aligned}
\int_{S_{1}}\left(G \delta_{i j}-\frac{\partial H}{\partial x_{j}}\left(y_{i}-\overline{x_{i}}\right) d S=\right. & \gamma\left(\mathbf{x}, S_{1}+S_{2}\right) \delta_{i j} \\
& -\int_{S_{2}}\left(G \delta_{i j}-\frac{\partial H}{\partial x_{j}}\left(y_{i}-\overline{x_{i}}\right) d S\right.
\end{aligned}
$$

where $S_{2}$ is any surface (not necessarily the remainder of the actual geometry) such that (a) $\mathrm{x}$ is not on either surface and (b) the composite surface $S_{1}+S_{2}$ is closed (i.e. encloses volume), as in the generic $2 \mathrm{D}$ Fig. 4(a). If $\mathbf{x}$ is not near to $S_{2}$, this converts the difficult integral over $S_{1}$ to a non-singular one over $S_{2}$. Comparable expressions apply to elasticity, with tensor operators but no essential analytic differences.

For non-crack points, $S_{2}$ may be taken to be all elements other than those actually incident to the singular point. For crack-surface points, this would include points on the 'far' crack surface, which are geometrically near the singular point, hence making the $S_{2}$ integral just as hard as the $S_{1}$ integral. This is 
remedied by constructing a temporary surface. Fig. 4(b) shows a construction for a $2 D$ crack surface, and Fig. 4(c) shows a ruled-surface closure over a 3D surface patch.

These expressions are a higher order analogy of the well known 'rigid body mode' arguments used in the primary BIE, hence the name 'modal method'.

These expressions are attractive because they (a) are extremely concise, (b) can be implemented using only repeated calls to existing integration routines for the integral over $S_{2}$, (c) are independent of the geometry of $S_{1}$ (i.e. apply when $S_{1}$ is curved and has corners), (d) apply in both $2 \mathrm{D}$ and $3 \mathrm{D}$, and (e) use only general reasoning, with no manipulation of the algebraic exprssions for the kernels.

An interior-to-exterior jump occurs in (9) when $\mathbf{x}$ crosses the surface and the value of $\gamma$ changes between 0 and 1 . The facts that the integrals (a) are finite and (b) approach fixed limits when $\mathbf{x} \rightarrow \overline{\mathbf{x}}$ from either direction are clear because all terms on the right are well behaved as long as $\bar{x}$ is not near the closure surface. One difficulty that is introduced is tha ${ }^{2}$ as $\overline{\mathbf{x}}$ is moved closer to the boundary of $S_{1}$ the integrals over $S_{2}$ become nearly-singular for some parts of $S_{2}$.

\subsection{Limit from the Interior}

The 'limit from the interior' process is an algebraic analysis with no exclusion zone. As in Fig. 5(a), $\mathbf{x}$ is considerd to approach a planar surface. At a (small but finite) distance from the surfaces, all kernels (hypersingular, Cauchy, and weaker, if desired) are written out in full and integrated analytically in the $\rho$ direction. Simple limit analysis at this point cancels all distance-to-surface terms. A jump between interior and exterior values of the Cauchy singularity (6) occurs due to ratios of signed and unsigned distance-to-surface terms at the time limits are taken.

The result of this process is a contour in tegral over the outer contour of the patch. This is well defined as long as that contour remains far from the singular point. ([GL90]) show that the process can be applied simultaneously to planar surfaces with a common edge at the singular point.

This process is used by ([GL90]), ([RB90]), and ([Gra89]) for the Laplace equation and by ([GMI90]) for elasticity.

\subsection{Exclusion Zone Methods}

Krishnasamy et al [KSRR90], Cruse et al ([CN90]) and Iokimidis et al [IP88] use algebraic methods with an exclusion zone Fig. 5(b). They are also known as (Hadamard) finite parts approaches. These are a higher-order analogy of Cauchy principal value methods. The key elements of these analyses are that (a) an exclusion zone is placed around the singular surface point, (b) the interior or exterior point $x$ approaches the surface faster than the exclusion zone vanishes. Arguments follow to show that integrals over the exclusion zone and the non- 
excluded part are all either finite or have infinite parts that are canceled by matching terms from the other surface portion.

A common requirement in these methods is that the distance between $\mathbf{x}$ and $\overline{\mathbf{x}}$ vanish faster than the size of the exclusion zone. Portions of ([KSRR90]) are identical to the limit-from-the interior process, with the rate-of-vanishing requirement forcing the exclusion zone to appear to be a finite size relative to the vanishing distance-to-surface The notation in ([CN90]) is diffrent because it is based on an indirect (displa ient discontinuity) form of the BIE. Their regularization is not done $a$ priori, but results from applications of Stokes' theorem and the divergence theorem to various individual terms.

All of these approaches defer the linear term (6) of the regularized expression for treatment by Cauchy principal value analysis. The jump between interior and exterior values occurs due to the free term of the Cauchy principal value analysis.

\subsection{Generalized Stokes Methods}

When a surface integrand can be shown to be the curl of another vector function, Stokes' theorem can be applied to convert a surface in tegral to a contour integral that is completely independent of the surface geometry. Fig. 3 has a branch for generalized application of this conversion. This is an incomplete branch of the tree.

([KSRR90]) gives an example of such conversion for the integral (5). The resulting vector expression is shown in Fig. 2. The corresponding conversion of the hypersingular elasticity $S_{i j k}$ kernel is given in ([Lut91]), along with additional cases for the Laplace equation. In principle, any integration done by the modal method can be done by a contour integral that is independent of the surface geometry. The only elasticity conversions known at present are the $S_{i j k}$ case just noted and the primary BIE Cauchy case given in ([GM87]).

\section{Conclusions}

We have discussed the common aspects of several approaches to computing the integrals in a hypersingular BIE. It is to be emphasized that the various results are fundamentally equivalent.

The modal methods are easy to work with if (a) the data structures in use permit construction of the necessary (temporary) closure surface for crack surface points and (b) integration procedures are accurate for near-singular integrals that often occur on the closure surface. ([Lut91]) demonstrates several examples of effective application of this method, including an example analysis of cracks in the US space shuttle fuel door hinge.

The methods grouped here as 'algebraic' present grueling analysis and coding tasks, particularly for curved surfaces. However, the analytic results may be highly efficient. 
Conversion to contour integrals via Stokes theorem is a very attractive operation when done in a generalized manner.

All methods are bound by the physical constraint that the integrations are well defined only when there is a $C^{1}$ interpolation of the solution function on the surface so that the Cartesian (rather than parametric) series expansions can be applied.

The analyses summarized in this paper have greatly improved our under standing of the behavior of the gradient BIE. These methods are now being used to model cracks and crack-like features. More broadiy, the rigors of the hypersingular case have contributed to a clearer understanding of the milder singularities in the primary BIE.

Acknowledgements: Financial support was provided by a fellowship from the Unisys Corporation. and by the Applied Mathematical Sciences Program, Office of Energy Research, US Department of Energy under contract DEAC05-84OR21400. Computer facilities were provided by the Cornell Fracture Group and Cornell University Program of Computer Graphics, funded by Dowell Schlumberger and the Digital Equipment Corporation.

\section{References}

[BB81] P.K. Banerjee and R. Butterfield. Boundary Element Methods in Engineering Science. McGraw Hill, 1981.

[BD88] C.A. Brebbia and X.Y. Dominguez. Boundary Elements: An Introductory Course. Computational Mechanics Publications (McGraw-Hill), 1988.

[BIL81] G.E. Blandford, A.R. Ingraffea, and J.A. Liggett. Two dimensional stress intensity factor computations using the boundary element method. International Journal for Numerical Methods in Engineering, 17:387-404, 1981.

[BTW84] C.A. Brebbia, J.C.F. Telles, and L.C. Wrobel. Boundary Element Techniques - Theory and Applications in Engineering. Springer-Verlag, 1984.

[Bui77] H.D. Bui. An integral equation for solving the problem of plane crark of arbitrary shape. J. Mech. Physics and Solids, 25:23-39, 1977.

[CN90] T.A. Cruse and G. Novati. Traction bie formulations and applications to non-planar and multiple cracks. In 22nd ASTM Conference on Fracture Mechanics. ASTM, 1990.

[Cru69] T.A. Cruse. Numerical solutions in three dimensional elastostatics. International Journal of Solids and Structures, 5:1259-1274, 1969.

[CVB71] T.A. Cruse and W. Van Buren. Three-dimensional elastic stress analysis of a fracture specimen with an edge crack. International Journal of Fracture Mechanics, 7:1-15, 1971.

[CZ52] A.P. Calderon and Zygmond. On the existence of certain singular integrals. Acta Mathematica, 88:85-139, 1952.

[GL90] L.J. Gray and E.D. Lutz. On the treatment of corners in the boundary element method. Journal of Computational and Applied Mathematics, 32:369-386, 1990. 
[GM87] N. Ghosh and S. Muhkerjee. A new boundary element method formulation for three dimensional problems in linear elasticity. Acta Mechanica, 67:107-119, 1987.

[GMI90] L.J. Gray, Luiz F. Martha, and A.R. Ingraffea. Hypersinguiar integrals in boundary element fracture analysis. International Journal for Numerical Methods in Engineering, 29:1135-1158, 1990.

[Gra89] L.J. Gray. Boundary element method for regions with thin internal cavities. Engineering Analysis, 6:180-184, 1989.

[IP88] N.I. loakimidis and M.S Pitta. Remarks on the gaussian quadrature rule for finite-part integrals with a second-order singularity. Computer Methods in Applied Mechanics and Engineering, 69:325-343, 1988.

[Jas63] M.A. Jaswon. Integral equation methods in potential theory, i. Proceedings of the Royal Society, 275:23-32, 1963.

[JSR90] Z.H. Jia, D.J.Shippy, and F.J. Rizzo. Boundary-element analysis of wave scattering from cracks. Communications in Applied Numerical Methods, 6:591601, November 1990.

[KSRR90] L.W. Krishnasamy, L.W. Schmerr, T.J. Rudolphi, and F.J. Rizzo. Hypersingular boundary integral equations: Some applications iti acoustic and elastic wave scattering. Journal of Applied Mechanics, 57:404-414, 1990.

[Kup65] V.D. Kupradze. Potential Methods in the Theory of Elasticity. D. Davey, 1965.

[Lut91] E.D. Lutz. Numerical Methods for Hypersingular and Near-Singular Boundary Integrals in Fracture Mechanics. PhD thesis, Cornell University, Ithaca, NY, USA, 1991.

[LW76] J.C. Lachet and J.O. Watson. Effictive numerical treatment of boundary integral equations: A formulation for three-dimensional elastostatics. International Journal for Numerical Methods in Engineering, 10:991-1005, 1976.

[Mik65] S.G. Mikhlin. Multidimensional Singular Integrals and Singular Integral Equations. Pergamon Press, 1965.

[MP80] S.G. Mikhlin and S. Proessdorf. Singular Integral Operators. Springer-Verlag, 1980.

[PCH87] E.Z. Polch, T.A. Cruse, and C.-J. Huang. Traction bie solutions for flat cracks. Computational Mechanics, 2:253-267, 1987.

[RB90] M. Rezayai and T. Burton. A boundary-integral formulation for complex three-dimensional geometries. International Journal for Numerical Methods in Engineering, 29:263-273, 1990.

[Riz69] F.J. Rizzo. An integral equation approach to boundary value problems of classical elastostatics. Quarterly of Applied Mathemathics, 25:83-95, 1969.

[Wea77] J. Weaver. Three dimensionial crack analysis. International Journal of Solids and Structures, 13:321-330, 1977. 

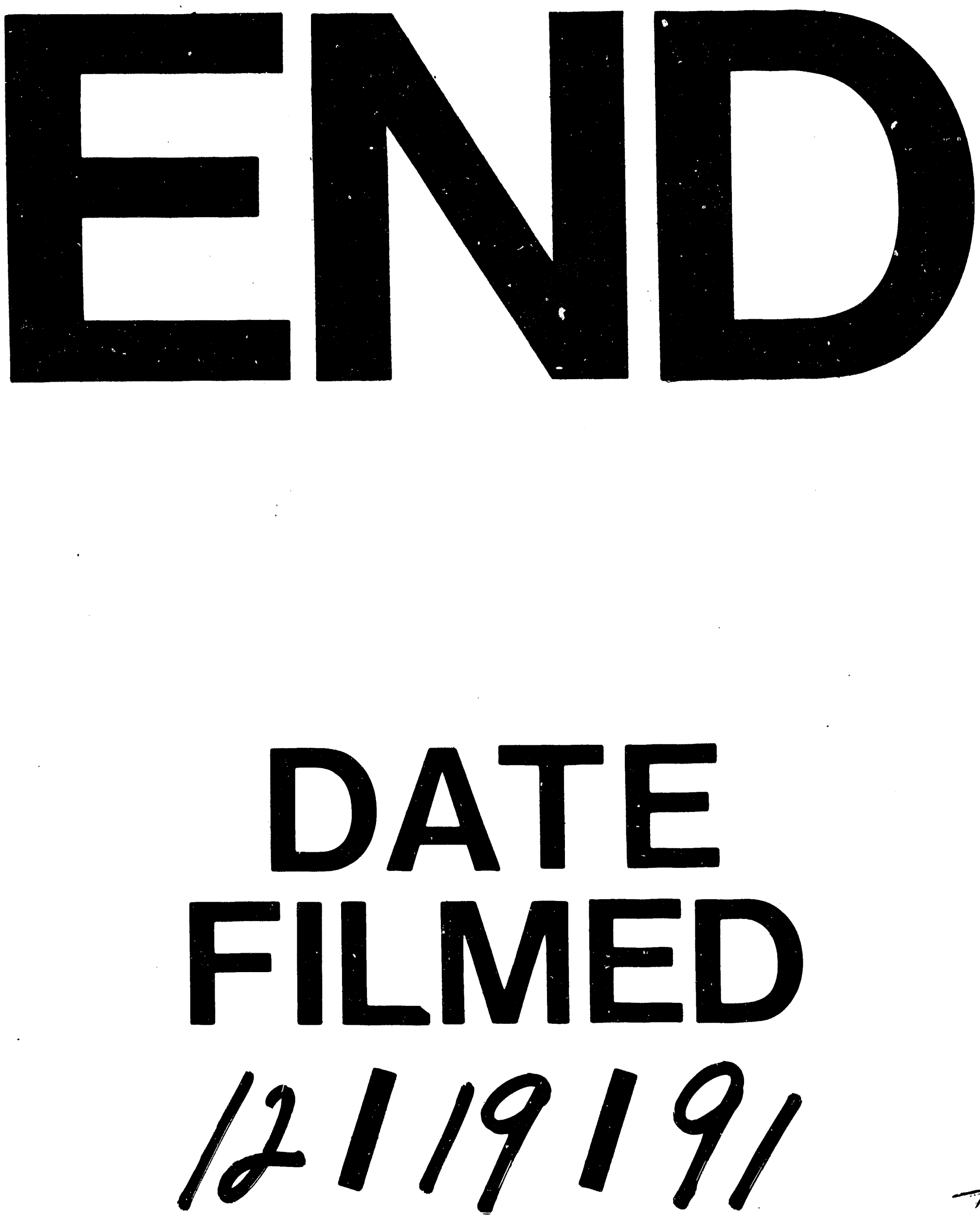

$\pi$ 